\title{
Neoplasias na microrregião de Vitória da Conquista, Bahia
}

Neoplasms in the microregion of Vitória da Conquista, Bahia

\author{
P.P. Almeida ${ }^{1}$; F.M.S. Lima ${ }^{1}$; P.R.L. Lacerda ${ }^{1}$; C.L. Macedo $^{2}$; G.S. Santos ${ }^{3}$; \\ R.A.A. Silva ${ }^{3 *}$ \\ ${ }^{1}$ Laboratório de Histopatologia, Universidade Federal da Bahia, Campus Anísio Teixeira, 45000-000, Vitória da \\ Conquista-BA, Brasil. \\ ${ }^{2}$ Serviço de Anatomia Patológica e Citopatologia - MICRO, 45020-290, Vitória da Conquista-BA, Brasil \\ ${ }^{3}$ Núcleo de Biointegração, Universidade Federal da Bahia, Campus Anísio Teixeira, 45000-000, Vitória da Conquista- \\ BA, Brasil.
}

*robson.amaro@gmail.com

(Recebido em 05 de outubro de 2015; aceito em 15 de dezembro de 2015)

\begin{abstract}
A incidência de neoplasias de mama, estômago e próstata têm aumentado consideravelmente no mundo, decorrente das mudanças no perfil de morbidade e mortalidade. Estudos acerca da ocorrência deste tipo de patologia fornecem informações sobre o perfil epidemiológico de determinada região, o que reflete diretamente a importância do estudo no controle e prevenção da doença, além do desenvolvimento de medidas para tratamento. Deste modo, o objetivo deste estudo foi analisar a ocorrência de neoplasias de mama, de próstata e de estômago, bem como caracterizar seu perfil histopatológico, na microrregião de Vitória da Conquista, Bahia. Foram analisados 3269 pacientes, correspondentes ao período de 2009 a 2013 , disponibilizados pelo Laboratório de Anatomia Patológica e Citopatologia MICRO, através da aplicação de um questionário para análise de variáveis como gênero, idade, ano da biópsia, neoplasia diagnosticada e seu subtipo histológico. Dentre as neoplasias investigadas, próstata mostrou-se mais ocorrente, correspondendo a $70,5 \%$ dos laudos analisados. Seguido de mama, que apresentou como subtipo histológico mais frequente o carcinoma ductal infiltrante. $\mathrm{O}$ adenocarcinoma gástrico do tipo intestinal foi o tipo histológico predominante encontrado na coleta dentre as neoplasias do estômago. Deste modo, o estudo fornece uma base acerca do perfil de ocorrência das neoplasias na microrregião de Vitória da Conquista, indicando que estas seguem os padrões epidemiólogicos relatados anteriormente para outras regiões.

Palavras-chave: neoplasias gástricas; neoplasias da próstata; carcinoma ductal.
\end{abstract}

The incidence of breast cancer, stomach and prostate cancers have increased considerably in the world, resulting from changes in the morbidity and mortality profile. Studies on the occurrence of this type of pathology provide information on the epidemiological profile of a given region, which directly reflects the importance of studying the control and prevention of disease, and the development of measures for treatment. Thus, the aim of this study was to analyze the occurrence of breast, prostate and stomach cancers as well as your histological profile in the microregion of Vitória da Conquista, Bahia. To do so, 3269 patients were analyzed, for the period 2009-2013, provided by the Laboratory of Pathology and Cytopathology MICRO, by applying a questionnaire to analyze variables such as gender, age, year of biopsy and its histological subtype. Among the investigated cancer, prostate was more occurring, corresponding to $70.5 \%$ of the analyzed reports. Followed by breast neoplasias, who presented the most common histological subtype infiltrating ductal carcinoma. Gastric adenocarcinoma intestinal type was the predominant histologic type found in the collection among the cancers of the stomach. Thus, the study provides a basis about the occurrence profile of cancers in the microregion of Vitória da Conquista, indicating that they follow the previously reported epidemiological patterns to other regions.

Keywords: gastric cancer; prostate cancer; ductal carcinoma. 


\section{INTRODUÇÃO}

A incidência de neoplasias aumentou consideravelmente nas últimas décadas no Brasil, reflexo de alterações ocorridas nos perfis de morbidade e mortalidade da população. Possivelmente justificada por um declínio na incidência e mortalidade de doenças infectocontagiosas e um aumento da ocorrência de doenças crônicas não transmissíveis, bem como da expectativa de vida da população [1]. As estatísticas [2] mostram que os tumores mais frequentes para o sexo masculino são os de próstata, pulmão, estômago, cólon e reto. Já para o sexo feminino predominam o câncer de pele, mama, seguidos das neoplasias de colo uterino, cólon e reto, pulmão e estômago.

A Organização Mundial da Saúde estimou [2] que em 2012 ocorreram 14,1 milhões de novos casos de câncer, sendo que destes, 8,2 milhões resultaram em óbito. No Brasil, de acordo com os dados do Instituto Nacional do Câncer (INCA), a estimativa para o ano de 2014 foi de aproximadamente 576 mil novos casos, sendo que na Bahia as neoplasias de próstata corresponderam a 3.450 mil dos novos casos estimados, mama feminina 2.560 mil e estômago 1.040 mil, ratificando a neoplasia como um grave problema de saúde pública [2].

A neoplasia maligna de mama é o segundo tipo de neoplasias mais frequente no mundo, e o primeiro mais comum entre as mulheres. Sua ocorrência gera impactos diretos na saúde emocional da mulher, comprometendo sua qualidade de vida ao tanger diversos aspectos como auto-estima, imagem corporal e sexualidade [3]. No sexo masculino, o câncer de prostáta apresenta-se como o mais frequente, sendo a realização do seu diagnóstico comprometida devido à tabus sociais construídos envolta do mesmo, alegando-se que sua realização compromete a virilidade e identidade masculina [4;5]. Além disso, esse gênero é mais acometido por neoplasias gástricas do que o feminino, destacando-se o adenocarcinoma gástrico como subtipo histológico mais comum, correspondendo à 95\% dos casos [6].

Alguns estudos demonstram que a urbanização da sociedade e as condições socioeconômicas são fatores consideráveis de risco para o câncer de mama [7], além do estilo de vida e a dieta também influenciarem significativamente. Embora estes dois últimos fatores tenham mais impacto na patogênese das neoplasias de origem gástrica, apresentam também influência no desenvolvimento das neoplasias prostáticas [8]. Além disso, fatores como o avançar da idade, a afrodescendência e a predisposição familiar aumentam a probabilidade do desenvolvimento destas últimas [9]. Alguns fatores ambientais têm forte associação com o desenvolvimento de neoplasias como: uma alimentação desequilibrada, exposição à ambientes insalubres, consumo de alguns medicamentos, hábitos e estilos de vida como o tabagismo, elitismo e sedentarismo $[9,10]$.

A neoplasia gástrica se encontra relacionada com a predisposição genética, como alterações no braço curto do cromossomo 7, os genes TP53 e TP73, e outras modificações que levam a desregulação do ciclo celular [11]. Possui forte relação principalmente com hábitos alimentares como ingestão de elevado teor de sal e aditivos alimentares, como nitrito, que ao ser metabolizado leva a formação de radicais livres que podem resultar em lesão e inflamação da mucosa gástrica [12]. Outro fator de risco para o desenvolvimento dessa patologia é a infecção por Helicobacter pylori. Alguns estudos salientam o papel desta bactéria no desenvolvimento do processo inflamatório com consequente indução de danos oxidativos, que podem estar relacionados à condições pré-neoplásicas [11].

$\mathrm{Na}$ histologia tumoral gástrica destaca-se a prevalência do adenocarcinoma gástrico (ACG), bastante frequente em homens com idade entre 50 e 70 anos [13]. Segundo a classificação de Lauren [14], o ACG pode ser dividido em dois tipos: o intestinal e o difuso. No ACG do tipo intestinal observa-se a presença de epitélio metaplásico, no qual o epitélio gástrico é substituído por elementos histológicos que reproduzem o epitélio duodenal. Esse tipo histológico se caracteriza pela presença de células neoplásicas coesas, formando estruturas tubulares e massa discreta. Já o ACG do tipo difuso é composto por células separadas ou em pequenos agrupamentos com secreção mucinosa e se infiltram difusamente na parede do estômago [6].

Relativo à histologia mamária, são identificados três padrões de lesões: alterações benignas, lesões malignas in situ e lesões malignas invasivas. As lesões malignas são denominadas carcinomas in situ e se referem à proliferação neoplásica de ductos e lóbulos limitada pela membrana basal. O tipo mais comum é o carcinoma ductal in situ, sendo seu subtipo arquitetural 
mais comum é o comedocarcinoma, caracterizado pela presença de massas sólidas de células pleomórficas e áreas de necrose. No carcinoma invasivo a membrana basal foi rompida e as células infiltraram o estroma mamário. $\mathrm{O}$ tipo histopatológico mais comum é o carcinoma ductal invasivo, ou infiltrante, seguido do carcinoma lobular invasivo [15].

O aumento da incidência e da mortalidade do câncer no Brasil reflete a necessidade de aperfeiçoamento do diagnóstico e acesso à terapia apropriada, bem como melhora da qualidade de informações acerca do mesmo [1]. A estratégia que se pode adotar para a diminuição da mortalidade pelo câncer de mama é o rastreamento. Este consiste em identificar o câncer em seus estágios iniciais quando geralmente é assintomático, possibilitando a mudança em seu prognóstico [16]. O aperfeiçoamento de métodos diagnósticos, ampliação do sistema de informação, promoção da saúde do homem, bem como o diagnóstico precoce demonstraram grande impacto nas estatísticas do câncer de próstata [2]. Métodos como PSA e a biópsia da próstata, permitem o reconhecimento de disfunções do tecido prostático em seus estágios iniciais, o que permite a reversão do quadro em seus momentos primários, como no caso da hiperplasia próstatica [4].

Os tumores selecionados para investigação são reflexo de uma preocupação protagonizada pela academia no seu compromisso de interação com a sociedade a qual encontra-se inserida, uma vez que os tumores em questão apresentam alta incidência nas demais regiões do país e possuem desconhecido perfil epidemiológico para a microrregião de Vitória da Conquista. Assim, o presente estudo teve como objetivo analisar a ocorrência de neoplasias da mama, de próstata e de estômago, bem como caraterizar o perfil histopatológico, na microrregião de Vitória da Conquista, Bahia.

\section{MATERIAL E MÉTODOS}

Trata-se de um estudo retrospectivo descritivo, onde foram analisados laudos de pacientes que se submeteram a exames anatomopatológicos no período de 2009 a 2013. Os laudos foram obtidos através do programa PathoControl (versão 8.0, Patho Control - Software para Laboratórios de Anatomia Patológica e Citopatologia, Natal, RN, Brasil) onde foi utilizada a base de dados do Laboratório de Anatomia Patológica e Citopatologia MICRO, responsável por atender cerca de $70 \%$ dos hospitais e clínicas localizados na microrregião de Vitória da Conquista.

Os tipos histológicos investigados foram adenocarcinoma da próstata, adenocarcinoma gástrico, carcinoma ductal infiltrante da mama, carcinoma lobular infiltrante da mama e carcinoma mucinoso da mama. Sendo as variáveis: gênero, idade, ano em que foi realizada a biópsia, a neoplasia diagnosticada seguida de seu subtipo histológico e a presença ou não de metástase. Os dados foram organizados em tabelas através do software Microsoft Office Excel 2013, onde foram calculados os percentuais. Os gráficos foram organizados com auxílio do software GraphPad Prism® (versão 5.03, GraphPad Software, San Diego, CA, USA), onde foi aplicado o teste exato de Fisher para analisar a correlação das variações histológicas com a idade e gênero relativos à histopatologia gástrica. As diferenças estatísticas foram consideradas significantes para valores com $\mathrm{p}<0,05 \mathrm{em}$ um intervalo de confiança de $95 \%$.

O presente estudo foi apreciado e aprovado pelo Comitê de Ética em Pesquisa envolvendo Seres Humanos do Instituto Multidisciplinar em Saúde, Campus Anísio Teixeira (IMS/CATUFBA), sob protocolo de $\mathrm{n}^{\mathrm{o}}$ 31075714.9.0000.5556.

\section{RESULTADOS}

Foram analisados 3269 laudos, correspondentes ao período de 2009 a 2013, onde todos foram diagnosticados com neoplasias, diferindo apenas o sítio anatômico. As neoplasias da próstata corresponderam a 70,5\% $(\mathrm{n}=2306)$ do total de laudos analisados, seguidos pela mama, $19,1 \%$ $(\mathrm{n}=625)$ e estômago, 10,3\% ( $\mathrm{n}=338)$.

Houve uma variação considerável nos números de casos por ano, porém foi observada uma relativa estabilidade na ocorrência das três neoplasias investigadas (Figura 1). Dentre as 
neoplasias da mama, observou-se maior ocorrência do subtipo histológico do Carcinoma Ductal Infiltrante da Mama (CDI), correspondendo a 86,7\% dos casos $(n=542)$, seguido do Carcinoma Lobular Infiltrante da Mama (CLI), 11,5\% ( $\mathrm{n}=72)$, e Carcinoma Mucinoso da Mama, 1,8\% $(n=11)$.

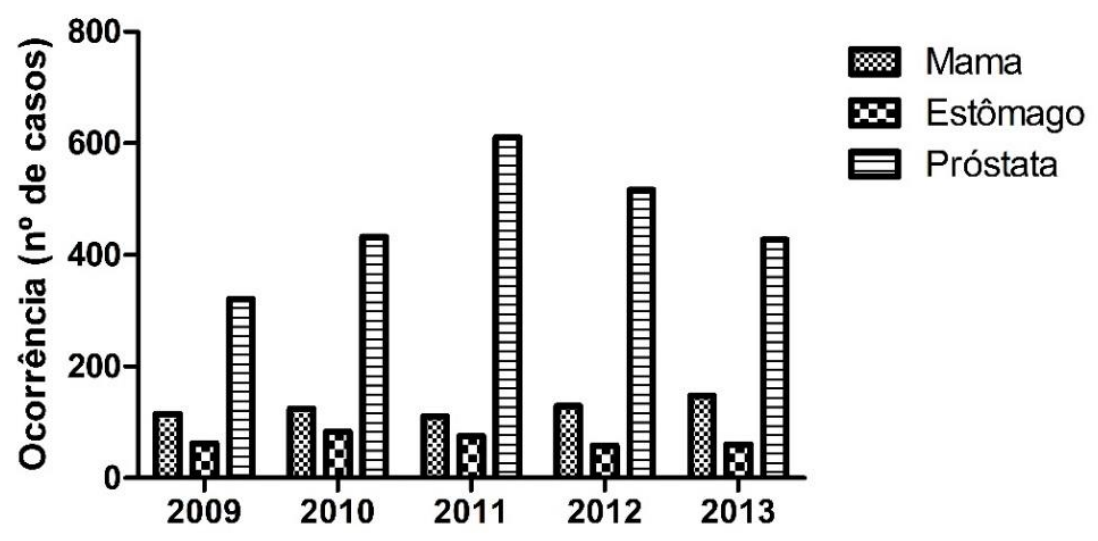

Figura 1. Distribuição temporal da ocorrência dos três tipos de neoplasias analisadas, no período de 2009 a $2013(n=3269)$.

A ocorrência dos carcinomas da mama se apresentou maior nas idades entre 40 - 69 anos, correspondendo a $66 \%$ da ocorrência total. Foi observada a presença de metástase em 14,4\% dos pacientes diagnosticados com câncer de mama.

O subtipo histológico predominante investigado de neoplasias da próstata foi $\mathrm{o}$ adenocarcinoma prostático (ACP), apresentando uma ocorrência na faixa etária de 60 - 89 anos $(n=1991)$. Observou-se ainda que em $78,1 \%$ dos laudos analisados, o ACP encontrava-se associado à outras disfunções do tecido da próstata, como hiperplasias, congestão em tecido adiposo e histiocitose em linfonodo ilíaco, sendo que a hiperplasia fibroadenomatosa (HFP) foi reportada em 58,1\% dos achados, seguida da hiperplasia nodular, 24,6\% (Tabela 1). Menos de $1 \%$ dos adenocarcinomas da próstata apresentou metástase.

\begin{tabular}{ccc} 
Tabela 1. Padrões histológicos associados ao & \multicolumn{3}{c}{ adenocarcinoma prostático } \\
\hline Disfunção & $\mathrm{n}$ & $\%$ \\
\hline Hiperplasia fibroadenomatosa da & 1048 & 58,1 \\
próstata & 444 & 24,6 \\
Hiperplasia nodular da próstata & 90 & 5,0 \\
Atrofia prostática & 222 & 12,3 \\
Outras $^{*}$ & 1804 & 100,0 \\
Total &
\end{tabular}

* Incluem congestões em tecido adiposo, muscular e vesículas seminal, histiocitose em linfonodo íliaco e prostatite crônica.

O câncer de estômago analisado foi o adenocarcinoma gástrico, sendo possível diferenciar os tipos difuso e o intestinal, acompanhados de lesão macroscópica do tipo ulcerada. A ulceração foi a lesão predominante encontrada. O tipo intestinal foi o que apresentou maior ocorrência, $53,8 \%$ (Tabela 2), destes 90,7\% estavam acompanhados de ulceração.

Ao se comparar a variação histológica do ACG segundo o sexo, observou-se que no sexo masculino, o tipo intestinal esteve presente em 59,07\% dos laudos, e o tipo difuso $27,8 \%$ dos laudos masculinos. No gênero feminino, o tipo intestinal representou 43,5\% dos laudos, sendo $40,59 \%$ correspondentes a esse tipo (Figura 2). Ao se comparar a variação histológica com o gênero, houve diferença estatisticamente significante $(\mathrm{p}=0,0106$, Odds ratio $=0,4980)$. 
Tabela 2. Diagnósticos histopatológicos de tumores gástricos nos laudos analisados no período de

\begin{tabular}{ccc} 
& 2009 a 2013. & \\
\hline Subtipo Histológico & $\mathbf{n}$ & $\mathbf{\%}$ \\
\hline ACG & 16 & 4,7 \\
ACG Ulcerado & 33 & 9,8 \\
ACG tipo Intestinal & 17 & 5,0 \\
ACG tipo Difuso & 27 & 8,0 \\
ACG Ulcerado Intestinal & 165 & 48,8 \\
ACG Ulcerado Difuso & 80 & 23,7 \\
Total & $\mathbf{3 3 8}$ & $\mathbf{1 0 0}$ \\
\hline
\end{tabular}

$\mathrm{ACG}=$ Adenocarcinoma Gástrico

A ocorrência segundo idade diferiu entre os dois gêneros, uma vez que as mulheres apresentaram o maior número de casos dos 60 - 69 anos, e os homens na faixa dos $70-79$ anos. Do total de pacientes analisados, $33 \%$ dos adenocarcinomas gástricos apresentaram metástase.

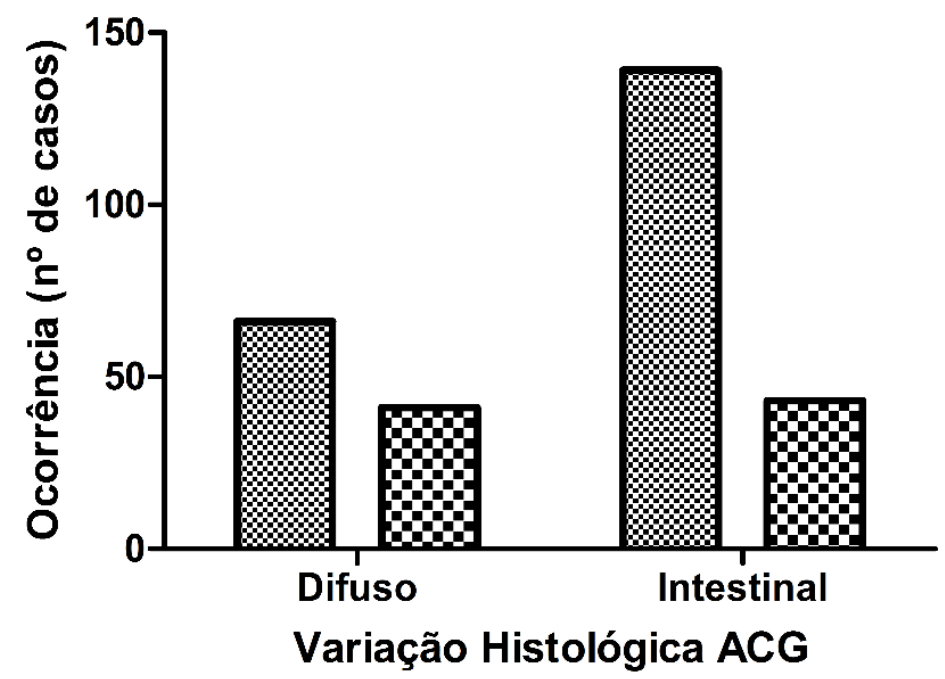

$\$$ Homem

Ex Mulher

Figura 2. Distribuição da variação histológica do Adenocarcinoma Gástrico (ACG) segundo o gênero, no período de 2009 a 2013 ( $n=338$ ), $p=0,0106$, Odds ratio $=0,4980$.

\section{DISCUSSÃO}

Neste estudo avaliou-se a ocorrência de neoplasias como próstata, mama e estômago na microrregião de Vitória da Conquista, cidade localizada no Sudoeste da Bahia, para avaliar se a situação da saúde da população nesta região segue o curso dos indicadores nacionais. Observouse que a ocorrência do câncer de mama apresentou discreto aumento ao longo dos anos, indicando comportamento epidemiológico similar ao estimado pelo INCA para os anos de 2010 e 2012 [17, $18]$.

Dentre seus subtipos histológicos, o carcinoma ductal invasivo (CDI) foi o mais ocorrente, seguido do carcinoma lobular invasivo e do carcinoma mucinoso, achados semelhantes aos de outros estudos $[19,20]$. A idade é apontada tendo um importante efeito prognóstico, sendo que a faixa etária de 40 - 49 anos apresenta melhores taxas de sobrevida, em comparação a mulheres acometidas antes dos 30 anos [19]. Em nosso estudo, evidenciou-se que $66 \%$ das mulheres acometidas com câncer de mama tinham idade entre 40 - 69 anos, sendo que a faixa etária entre 50 - 59 anos obteve o maior número de casos $(25,6 \%)$, dado relatado anteriormente na literatura para outras regiões [20].

O CDI apresentou discreto aumento na ocorrência ao longo dos anos analisados, tendo maior ocorrência em 2013, onde foram observados 136 pacientes com esse tipo histológico. Em contrapartida, o CLI apresentou diminuição no número de casos, chegando a apenas 9 casos 
$(1,44 \%)$ em 2013. A associação das variações histológicas com a taxa de sobrevida é contraditória. Alguns estudos relatam que a histologia não se relaciona com a curva de sobrevida dos pacientes [21, 22], entretanto, a variação histológica tumoral pode estar diretamente associada com a geração de metástases, sendo que os carcinomas in situ apresentam melhores prognósticos, não apresentando capacidade de invasão tecidual, a priori [23]. Além disso, tem sido mostrado que o CDI é relatado como o subtipo com maior envolvimento linfático, apresentando as maiores taxas de mortalidade e grande propensão à invasão tecidual [23, 24]. Em nosso estudo, onde $87,8 \%$ dos carcinomas metastáticos corresponderam ao CDI.

O câncer de próstata tem se caracterizado como o tipo mais incidente na população masculina e pode ser justificado pelo caráter silencioso da doença. Por apresentar características apenas nas fases mais avançadas, há uma dificuldade no diagnóstico precoce, que poderia identificar o crescimento da glândula ainda na fase de hiperplasia benigna da próstata, permitindo o tratamento antes do agravo dos sintomas e consequente evolução maligna [3]. Em nosso estudo observamos que $82,7 \%$ dos pacientes analisados cursam com adenocarcinoma da próstata, também apresentam hiperplasia fibroadenomatosa da próstata ou hiperplasia nodular da próstata associada.

A hiperplasia é apontada [25] como mais prevalente que o próprio câncer de próstata, podendo apresentar sérias complicações pelo caráter progressivo da doença sob efeito dos androgênios, apresentando-se mais ocorrente em homens com faixa etária mais avançada.

Um estudo realizado por Nassif (2009) [26] demonstrou que a maior incidência de desenvolvimento da doença é na sexta década de vida, oscilando na faixa etária de 50 - 79 anos, apontando a idade como um dos fatores determinantes para o desenvolvimento do câncer de próstata. Dessa forma, nossos dados corroboram com os achados de outros autores em que a faixa etária mais acometida pela neoplasia compreende idades entre 60 - 79 anos. Destacamos também a ocorrência de casos na faixa etária entre 40 - 49 anos que podem ser justificados pelo aprimoramento e popularização das técnicas de detecção em fases iniciais da doença [27].

A neoplasia da próstata é uma patologia cujo agravo está diretamente associado ao diagnóstico tardio ou ausência de tratamento. Sabendo que os homens adentram o sistema de saúde por meio da atenção especializada, torna-se necessário o desenvolvimento de programas na atenção primária visando prevenir agravos evitáveis [28]. Sendo assim, o INCA, órgão do ministério da saúde responsável por efetivar políticas prevenção e controle da doença, tem desenvolvido estratégias que objetivam a promoção da saúde, detecção precoce, diagnóstico e tratamento, bem como cuidados paliativos para os indivíduos que se encontram na fase avançada da doença. Com a finalidade de assistir esses pacientes nas diferentes fases evolutivas desta afecção, aumentando as chances de cura e diminuindo os índices de mortalidade [29].

No adenocarcinoma gástrico (ACG) pode se observar duas variações histológicas, o ACG Difuso e o ACG Intestinal, podendo apresentar lesões associadas [14]. Em estudo feito por Arregi e cols (2009) [13], foi observado que as variações apresentavam-se mais ocorrentes no sexo masculino, indicando ainda uma diferença na prevalência do subtipo histológico por gênero, onde nos homens foi mais frequente o tipo intestinal, estando essa variação mais propensa a ocorrer em neste sexo, como também demonstrado em nosso estudo.

A gastrite crônica atrófica e a ulceração são apontadas como fatores preditivos para a etiologia tumoral gástrica, uma vez que podem se encontrar associados com a metaplasia intestinal, processo caracterizado pela presença de um epitélio com características morfológicas e bioquímicas do epitélio intestinal, podendo comprometer a funcionalidade do órgão $[8,30]$. A presença de ulceração acompanhou $83,3 \%$ dos ACGs, sendo que lesão estava associada principalmente ao tipo intestinal, correspondendo a $48,8 \%$ dos laudos.

O sexo masculino é o mais acometido com ACG, principalmente tipo intestinal [31], podendo estar relacionando à sua maior susceptibilidade a adoção de hábitos não saudáveis como tabagismo, etilismo e alimentação rica em gordura e conservantes que podem agredir a mucosa gástrica. Nosso estudo demonstrou uma ocorrência de 69\% do ACG no sexo masculino, dado também encontrado por Lemes e cols (2003) [31], e ainda demonstram que a faixa etária mais comum de ocorrência das neoplasias gástricas é entre 50 - 79 anos, sendo a incidência deste tipo de neoplasia em jovens menos frequente, podendo chegar a cerca de 5\% do total de casos [33], corroborando os dados do nosso estudo. 
As metástases linfonodais são comuns nas neoplasias gástricas, sendo rara a sua incidência em tumores menores do que $2 \mathrm{~cm}$, do tipo I (elevados), bem diferenciados, do tipo intestinal de Lauren e restritos à mucosa [34]. Das três neoplasias investigadas, o ACG foi o que mais gerou metástases, ficando em segundo lugar os carcinomas mamários.

\section{CONCLUSÃO}

No presente estudo observou-se que as neoplasias de mama, próstata e estômago analisadas seguem os padrões epidemiológicos relatados para outras regiões do país. Dentre as cidades citadas anteriormente, assemelham-se a nossos estudos, o Rio de Janeiro, demonstrando estudos relativos ao câncer de mama, bem como o Ceará, relativo a tumores gástricos, e Rio Grande do Sul, relativo ao câncer de próstata, com apresentação acerca das características epidemiológicas e demográficas dos tumores citados. Além disso, as estimativas realizadas pelo INCA para o número de novos casos de câncer, nos anos de 2012 e 2014, apontam os tumores da mama e da próstata como os mais estimados nos três estados citados, ficando os tumores gástricos em terceiro lugar para o Rio de Janeiro e Ceará. Desse modo, a avaliação da ocorrência destas patologias na região poderá auxiliar no planejamento e aprimoramento de ações que fomentam estratégias que visam a prevenção, o tratamento e disponibilidade de informações, bem como promovam e assegurem atenção à saúde de forma eficiente e qualificada.

\section{REFERÊNCIAS BIBLIOGRÁFICAS}

1. Boing AF, Vargas, SAL, Boing AC. A carga das neoplasias no Brasil: mortalidade e morbidade hospitalar entre 2002-2004. Rev Assoc Med Bras. 2007; 53(4): 317-22.

2. BRASIL. Ministério da Saúde. Estimativas 2014: Incidência de Câncer no Brasil. Rio de Janeiro: Ministério da Saúde, 2014.

3. Maluf, MFM, Mori LJ, Barros ACSD. O impacto psicológico do câncer de mama. Revista Brasileira de Cancerologia. 2005;. 51(2): 149-154.

4. Tonon TCA, Schoffen JPF. Câncer de Próstata: uma revisão da literatura. Revista Saúde e Pesquisa. 2009; 2(3): 403-10.

5. Soares DAS. Câncer de próstata: as barreiras para a realização do toque retal [trabalho de conclusão de curso]. Governador Valadares (MG): Universidade Federal de Minas Gerais; 2014. 26p.

6. Vinagre RMDF, Campos BP, Sousa RMP. Case study of stomach adenocarcinoma conducted at a cancer referral hospital in northern Brazil. Arq. Gastroenterol. 2012; 49(2): 125-29.

7. Torio CM, Klassen AC, Curriero FC, Caballero B, Helzlsouer K. The modifying effect of social class on the relationship between body mass index and breast cancer incidence. Am J Public Health. In press 2009.

8. Epstein JI, Amin M, Boccon-Gibod L, Egevad L, Humphrey PA, Mikuz G, et al. Prognostic factors and reporting of prostate carcinoma in radical prostatectomy and pelvic lymphadenectomy specimens. Rev Scand J Urol Nephrol Suppl 2005; 216:34-63.

9. Gonçalves IR, Padovani C, Popim RC. Caracterização epidemiológica e demográfica de homens com câncer de próstata. Ciências \& Saúde Coletiva. São Paulo; 2008; 13(4): 1337-42.

10. Medeiros APD, Menezes MDFBD, Napoleão AA. Fatores de risco e medidas de prevenção do câncer de próstata: subsídios para a enfermagem. Rev. bras. Enferm. 2001; 64(2): 385-88.

11. César ACG, Silva AE, Tajara EH. Fatores genéticos e ambientais envolvidos na carcinogênese gástrica. Arq Gastroenterol. São Paulo; 2002; 39(4): 253-359.

12. Garófolo A, Avesani CM, Camargo KG, Barros ME, Silva SRJ, Taddei JAAC, et al. Diet and cancer: an epidemiological view. Revista de Nutrição. Campinas. 2004. 17(4): 491-505.

13. Arregi MMU, Ferrér DPC, Assis ECV, Paiva FDS, Sobral LBG, André NF, et al. Perfil ClinicoEpidemiológico das Neoplasias de Estomago Atendidas no de Câncer do Instituto do Câncer do Ceará no Periodo de 2000-2004. Revista Brasileira de Cancerologia. Ceará; 2009; 2(55): 121-28.

14. Lauren P. The two histological types of gastric carcinoma: diffuse and so-called intestinal-type carcinoma. An attempt at a histoclinical classification. Acta Pathol Microbiol Scand. 1965; 64: 31-49.

15. Kumar V, Abbas AK, Fausto N. Robbins \& Cotran: Patologia -Bases Patológicas das Doenças. $7^{\text {a }}$ Ed. Rio de Janeiro: Elsevier, 2004.

16. Thuler LC. Considerações sobre a prevenção do câncer de mama feminino. Revista Brasileira de Cancerologia. 2003; 49(4): 227-38. 
17. Instituto Nacional do Câncer. Encarte especial. Estimativas 2010: Incidência de Câncer no Brasil. Rio de Janeiro: Ministério da Saúde, 2010.

18. BRASIL. Ministério da Saúde. Estimativas 2012: Incidência de Câncer no Brasil. Rio de Janeiro: Ministério da Saúde, 2012.

19. Abreu E, Koifman S. Fatores prognósticos no câncer da mama feminina. Revista Brasileira de Cancerologia. 2002; 48(1): 113-31.

20. Martins E, Junior RF, Curado MP, Freitas NMA, Oliveira JC, Silva CMB. Evolução temporal dos estádios do câncer de mama ao diagnóstico em um registro de base populacional no Brasil Central. Rev Bras Ginecol Obstet. Goiânia; 2009; 31(5): 219-23.

21. Schneider IJC, D’orsi E. Sobrevida em cinco anos e fatores prognósticos em mulheres com câncer de mama em Santa Catarina, Brasil. Cad. Saúde Pública. Rio de Janeiro; 2009; 25(6): 1285-96.

22. Moraes AB, Zanini RR, Turchiello MS, Riboldi J, Medeiros LR. Estudo da sobrevida de pacientes com câncer de mama atendidas no hospital da Universidade Federal de Santa Maria, Rio Grande do Sul, Brasil. Cad. Saúde Pública. Rio de Janeiro; 2006; 22(10): 2219-28.

23. Cambruzzi1 E, Zettler CG, Pêgas KL, Duarte MR, Candiota JS, Teixeira SL. Associação entre metástases em linfonodos axilares e fatores prognósticos e preditivos no carcinoma ductal infiltrante de mama. Revista da AMRIGS. Porto Alegre; 2010; 54 (3): 294-99.

24. Cintra JRD, Teixeira MTB, Diniz RW, Junior HG, Florentino TM, Freitas GF, et al. Perfil imunohistoquímico e variáveis clinicopatológicas no câncer de mama. Rev Assoc Med Bras. Juiz de Fora; 2012; 58(2): $178-87$.

25. Averbeck MA, Blaya R, Seben RR, Lima NG, Denardin D, Fornari A, Rhoden EL. Diagnóstico e tratamento da hiperplasia benigna da próstata. Revista da AMRIGS, 2010; 54(4): 471-77.

26. Nassif AE, Filho RT, Paula RXG, Taguchi WS, Pozzobon HJ. Perfil epidemiológico e fatores prognósticos no tratamento cirúrgico do adenocarcinoma de próstata clinicamente localizado. Rev. Col. Bras. Rio de Janeiro; 2009; 36(4): 327-33.

27. Gomes R, Rebello LEFS, Araújo FC, NascimentoEF. A prevenção do câncer de próstata: Uma revisão da Literatura. Ciência \& Saúde Coletiva. Rio de Janeiro; 2008; 13(1): 235-246.

28. BRASIL, Ministério da Saúde. Política Nacional de Atenção Integral a Saúde do Homem (Princípios e Diretrizes). Brasília: Ministério da Saúde, 2008.

29. BRASIL, Ministério da Saúde. Programa Nacional de Controle do Câncer de Próstata. Rio de Janeiro: INCA, 2002.

30. Rodrigues LGM, Nogueira AMMF, Araújo LA, Salles PGO, Carvalho SPD, Cabral MMDA. Metaplasia intestinal e carcinoma gástrico: correlação com os subtipos histológicos da neoplasia. $\mathrm{J} \mathrm{Br}$ Patol. 2001; 37(4): 279-86.

31. Abib AR, Oliveira IM, Koifman S. Histopatologia do câncer de estômago (classificação de Lauren) em amostra de pacientes hospitalares no Rio de Janeiro, 1980-1995. Cad. Saúde Públ. Rio de Janeiro; 1997; 13(1): 99-104.

32. Lemes LA, Neunschwander LC, Matta LA, Osório-Filho J, Soares PCM, Cabral MMDA et al.. Carcinoma gástrico: análise sistemática de 289 gastrectomias consecutivas em Belo Horizonte (MG). J Bras Patol Med Lab. 2003; 39(1), 57-65.

33. Mauad EC, Nogueira JL, Souza JMP, Wohnrath DR, Oliveira ATT, Colli G, et al. Câncer Gástrico em Adultos Jovens. Revista Brasileira de Cancerologia. São Paulo; 2000; 3(46): 299-304.

34. Neto GPB, Santos EG, Victer FC, Carvalho CES. Metástase linfonodal em câncer gástrico precoce. Rev. Col. Bras. Cir. Rio de Janeiro; 2014; 41(1): 011-017. 